\title{
CONTRIBUCIÓN DE LA FACULTAD DE FARMACIA DE SANTIAGO DE COMPOSTELA AL DESARROLLO DE LA INVESTIGACIÓN CIENTÍFICA ESPAÑOLA EN EL PRIMER TERCIO DEL SIGLO XX (1900-1936)
}

\section{Beatriz Brasa Arias, Santiago Sanmartín Míguez y Mariana Landín}

Universidad de Santiago de Compostela

\section{RESUMEN}

La Facultad de Farmacia de Santiago de Compostela se fundó en 1857, pero no fue hasta comienzos del siglo XX cuando comenzó a desarrollar una labor de investigación en medicamentos. Las nuevas reglamentaciones que fomentaban la experimentación en los laboratorios universitarios y la formación de profesores y recién licenciados en el extranjero, contribuyeron a generar un grupo investigador notable dentro de una Facultad de provincias. La excesiva centralización en materia universitaria de la época y las características de la sede de la Facultad —un palacio renacentista del siglo XVI- dificultaron, en general, el desarrollo de la investigación. Sin embargo, el espíritu científico de profesores y alumnos, y su trabajo se impusieron y lograron un estatus digno en investigación de medicamentos en Galicia. En estos años comenzaron a publicarse trabajos originales principalmente en la «Revista de Farmacia», publicada por la propia Universidad compostelana.

En los años precedentes a la guerra civil española (1936) el nivel alcanzado en investigación y desarrollo de medicamentos era importante, favoreciendo el hecho de que, una vez comenzada la contienda, el personal de la Facultad trabajó en el laboratorio de farmacia militar en el que fue transformada por el ejército franquista. Durante este período la labor investigadora continuó, aunque a menor escala, y orientada a la obtención de «medicamentos copia» de otros específicos que escaseaban en tiempos de guerra ${ }^{1}$. Durante el primer tercio del siglo XX se desarrolló el germen del prestigio investigador del que goza en la actualidad la Facultad de Farmacia compostelana.

PALABRAS CLAVE: Galicia. Facultad de farmacia. Santiago de Compostela. Investigación científica. Desarrollo de medicamentos. Revista de Farmacia.

1 Brasa Arias, B. (2007), Siglos XX y XXI. En Sanmartín MigueZ, S. (coord), De Pharmaceutica Scientia. 150 años de la facultad de farmacia (1857-2007), Santiago de Compostela, Ed. Universidade de Santiago, p. 200. 


\title{
CONTRIBUTION OF THE FACULTY OF PHARMACY OF SANTIAGO DE COMPOSTELA TO THE DEVELOPMENT OF THE SPANISH SCIENTIFIC RESEARCH IN THE FIRST THIRD OF THE 20TH CENTURY (1900-1936)
}

\begin{abstract}
The Faculty of Pharmacy of Santiago de Compostela was founded in 1857, but research activities did not start until the beginning of the 20th century. The new Spanish regulations promoting the experimentation at universities and the stages of professors and recently graduated students outside Spain contributed to generate a remarkable research group in a provincial university. The excessive university centralism at that time in Spain and the characteristics of the headquarters of the faculty - a 16th century Renaissance palace - hindered, in general, the research development. However, the scientific spirit of professors and students, and their work were imposed and they achieved an estimable status in pharmacy research in Galicia. The facilities were the staff's highest priority and it raised more than a protest. A remarkable investment in apparatuses and laboratory materials was carried out and researchers started to publish original research papers in local journals, such as the «Revista de farmacia», published by their own University.

In the previous years to the Spanish Civil War (1936) the research status in drug development and the background of the staff and students were important, allowing them to join the military laboratory that Franco's army created at the faculty of pharmacy. The research work continued in a small scale and was specially focused on the production of «copy drugs» copying European specific drugs which were scarce at that time. The first third of the 20th century was the germ of the great research prestige that the Faculty of Pharmacy of Santiago de Compostela enjoys at present.
\end{abstract}

KEY WORDS: Galicia. Faculty of Pharmacy. University of Santiago. Science research. Drug development. Revista de Farmacia.

\section{INTRODUCCIÓN}

Durante el primer tercio del siglo XX, fructificaron en España las reformas iniciadas desde la Ilustración y desarrolladas a lo largo del siglo XIX, dirigidas a conseguir una enseñanza experimental y un mayor nivel investigador ${ }^{2}, \mathrm{y}$ el país vivió la denominada «Edad de Plata de la Ciencia española» cercenada por la guerra civil de 1936.

Las nuevas disposiciones gubernamentales fueron claves para la modernización de la Universidad compostelana particularmente anquilosada debido a la férrea influencia del clero $^{3}$. Las facultades experimentales (Medicina,

2 Acerca de las reformas en la universidad española desde la Ilustración, véase PESET, M. y PESET, J.L. (1974), La Universidad española: (siglos XVIII y XIX): despotismo ilustrado y revolución liberal, Madrid, Taurus.

3 Para completar la historiografía sobre la Universidad de Santiago de Compostela, véanse VV.AA. (1995) Gallaecia Fulget: (1495-1995): cinco séculos de historia universita- 
Ciencias y Farmacia) comenzaron una profusa labor investigadora que pronto se tradujo en una mayor producción científica ${ }^{4}$.

Este trabajo pretende dejar constancia de la interesante labor investigadora realizada en este periodo en la facultad de Farmacia de la Universidad compostelana, consecuencia directa de unas acertadas políticas gubernamentales y del interés y el esfuerzo personal de sus propios miembros.

\section{LA SEDE DE LA FACULTAD DE FARMACIA}

La Ley de 9 de septiembre de 1857, elaborada por el Ministro de Instrucción Pública Claudio Moyano, dictaminó la creación de la Facultad de Farmacia de Santiago de Compostela que, de esta manera, se sumó a las tres que previamente existían en el país: Madrid, Barcelona y Granada. La nueva Facultad se estableció en el colegio de Fonseca, un palacio del siglo XVI. Este edificio fue compartido con la Facultad de Medicina hasta que esta última se trasladó a sus nuevas dependencias en el año 1928.

Durante los más de cien años que fue sede de la Facultad de Farmacia su estructura interna fue muy poco modificada, hecho perfectamente comprensible si tenemos en cuenta el elevado coste económico y la difícil labor que suponen realizar obras en un edificio renacentista construido en su totalidad con piedra de granito. Las crónicas de esta época hablan de las incomodidades que el colegio de Fonseca presentaba para la vida cotidiana: humedad, falta de gas, calefacción de carbón insuficiente para calentar los amplios espacios delimitados por centenarios muros de piedra, laboratorios pequeños $\mathrm{y}$ oscuros, etc. ${ }^{5,6}$

ria, Santiago de Compostela, Ed. Universidade de Santiago de Compostela; y SÁnchez BARREIRO, X.R. (coord.) (2003) Historia de la Universidad de Santiago de Compostela, Santiago de Compostela, Universidade de Santiago de Compostela, Servicio de Publicacións e Intercambio Científico

4 Para mayor información sobre esta época de la Universidad de Santiago de Compostela consultar a BARREIRO FERNÁNDEZ, X.R. (1980), «La Universidad gallega entre 1900 y 1936», en: M. C. Díaz y Díaz (coord.) La Universidad de Santiago, Santiago de Compostela, Universidad, Secretariado de Publicaciones, pp. 77-87; y GURRIARÁN RODRÍGUEZ, R. (2006) Ciencia e conciencia na Universidade de Santiago (1900-1940): do influxo institucionalista e a JAE á depuración do profesorado, Santiago de Compostela, Universidad, Secretariado de Publicacións e Intercambio Científico.

5 Eleizegui López, A. (1906) La reorganización de los estudios de Farmacia. Discurso inaugural del curso 1906-1907, Santiago de Compostela. 
A lo largo de las primeras décadas del siglo XX, se abordaron algunas obras de remodelación y acondicionamiento dirigidas, en su mayor parte, a dotarlo de las características mínimas adecuadas para el desarrollo de una tarea investigadora, que han quedado recogidas en el libro de actas de la facultad. Por ejemplo, en la sesión de 9 de septiembre de $1922^{7}$ puede leerse:

«Para proseguir esta labor de arreglo de locales y Laboratorios de Químicas y Materia Farmacéutica Vegetal se precisan 20.000 pesetas con destino a estos para construcción de mesas de trabajo, armarios, conducción de aguas y gas, y material ordinario, además de considerar necesarias unas 800 pesetas para mantenimiento de la calefacción en los citados laboratorios».

Se adjunta al acta un presupuesto para construir un nuevo laboratorio, en el ángulo sudeste del piso principal del edificio, dentro de la cátedra de Materia Farmacéutica Vegetal - «el más económico de los que se han presentado al pedirlo el que suscribe a los más acreditados maestros de obras de Santiago»- que ilustra acerca del estado del edificio donde tenían lugar las labores de docencia e investigación y las necesidades inmediatas:

- Asfaltado de las paredes, cuya permeabilidad haría imposible la conservación del material que allí ha de instalarse .................... 300 ptas

— Enlucido de techos y paredes .................................................. 750 ptas

- Armario de 3 metros de alto y cuatro de ancho para guardar el material 800 ptas

- Instalación de luz eléctrica 150 ptas

- Dos mesas para trabajo y una para la instalación de microfotografía 317 ptas

Con presupuestos reducidos, un total de 2.317 pesetas en este caso, y con proyectos de remodelación poco definidos, Fonseca fue viendo reconstruidas o acondicionadas sus instalaciones en un intento de adaptarse a los nuevos tiempos. En una nota suelta ${ }^{8}$ sin fechar pero probablemente escrita entre los años 1921 y 1924 aparecen las medidas de una parte del edificio, junto con

6 Cuenca AraúJo, E. (1918) Renovación de la profesión farmacéutica en sus relaciones con la enseñanza universitaria. Discurso inaugural del curso 1918-1919, Santiago de Compostela.

7 Libro de Actas de la facultad de farmacia de Santiago (1915-1936). Depositado en el Decanato de la facultad.

8 Archivo Histórico Universitario de Santiago. Facultades. Otros papeles de Farmacia. Leg. 348 
algunos comentarios anotados al margen: «Hay que dar paso interior para todas estas habitaciones que forman dos pabellones independientes» $\mathrm{y}$ «Ver si se puede poner un retrete en la obra nueva». Resulta arriesgado hacer una valoración de una simple nota, pero la información sugiere que las reformas se iban planificando sobre la marcha.

El traslado de la Facultad de Medicina a un nuevo edificio en 1928, proporcionó a los profesores de Farmacia un mayor espacio para desarrollar sus tareas docentes e investigadoras y se abordaron reformas para dotar al Colegio de Fonseca de nuevas dependencias que permitieran comenzar a impartir clases prácticas, aspecto que hasta la fecha no había tenido la debida importancia en la Facultad. En enero de 1933 se publicó un artículo en la Revista de Farmacia 9 en el que se daba cuenta de las obras realizadas y la dotación material de los nuevos laboratorios de Química Inorgánica, Química Analítica y Técnica Física.

La descripción que el autor hace de uno de los laboratorios proporciona una idea de las carencias sufridas hasta el momento ya que, como puede observarse en los párrafos siguientes, se hace especial referencia a la acometida de electricidad, agua y gas, aspectos que son imprescindibles para el desarrollo de cualquier tarea investigadora.

«el laboratorio de Química Inorgánica destinado a los alumnos, se compone de una amplia sala, con tres mesas de 3x 1,40 m. y de 0,90 de altura. En cada mesa hay una estantería de tres pisos, para los reactivos, 8 tomas de gas, 8 grifos para agua y dos piletas centrales para desagüe. El sistema de desagüe consta además de una pila rectangular, colocada en uno de los extremos: en la pila van tres grifos de agua, uno para lavado de aparatos de gran longitud y dos de tipo corriente, para la instalación de trompas. La energía eléctrica se distribuye mediante dos enchufes. El tablero de la mesa se ha impregnado de negro de anilina, preparado sobre la misma mesa [...]. En los dos lienzos de pared entre las ventanas centrales, se colocaron poyatas de $0,60 \mathrm{~m}$. de ancho, con servicios de gas, agua y energía eléctrica, que permiten un desahogo en el trabajo de las mesas. [...] Completan la instalación: un amplio armario destinado para almacén de material y productos químicos de uso frecuente.»

Las obras fueron financiadas por el Patronato Universitario de la Universidad de Santiago, que por aquel entonces también tenía prevista la construcción de la cátedra y laboratorio de Química Orgánica, y que ya había subven-

9 ACHA (1933), Los nuevos laboratorios de Química Inorgánica y Analítica de la Facultad de Farmacia de Santiago, Revista de Farmacia, 12 (1), pp. 1-7. 
cionado anteriormente los laboratorios de Botánica y Mineralogía, así como un museo dedicado a ambas asignaturas ${ }^{10}$.

\section{LOS ESTUDIOS DE FARMACIA Y LA INVESTIGACIÓN CIENTÍFICA}

El siglo XX comenzó con un nuevo plan de estudios para las facultades de Farmacia. Si bien el anterior plan de $1886^{11}$ había hecho hincapié en la importancia de la enseñanza experimental, el recién estrenado de $1900^{12}$ incrementaba de forma notable el número de horas de prácticas de laboratorio y las excursiones científicas, lo que contribuyó positivamente al desarrollo de la investigación en la Facultad. La introducción de materias de carácter experimental o que, incluso en su nombre, llevaban escrito el «junto con sus prácticas», suscitaron el interés del colectivo universitario por el trabajo de laboratorio.

Después de un año de preparatorio en la Facultad de Ciencias los alumnos cursaban en cuatro años: Técnica Física, Mineralogía y Zoología, Botánica descriptiva, Química Inorgánica, Materia Farmacéutica Vegetal, Química Orgánica, Análisis Químico de alimentos, medicamentos y venenos y Farmacia Práctica y Legislación.

A pesar de que los primeros años del siglo XX constituyeron la época dorada de la Microbiología y otras ciencias biológicas como consecuencia de los trabajos de Louis Pasteur, Robert Koch, Alexander Fleming o Ramón y Cajal, que revolucionaron la Medicina y las ciencias farmacéuticas, estas materias no se introdujeron inmediatamente en los estudios de las universidades periféricas (Barcelona, Granada y Santiago) sino que formaban parte del doctorado que solo podían cursarse en la Universidad Central en Madrid.

De esta forma, la reglamentación en materia de contenidos docentes constituyó el primer impulso hacia la consolidación de la investigación científica en Fonseca aunque supuso también una limitación para el desarrollo de otras florecientes ciencias, como la Microbiología, que no pudieron desarrollarse en la Facultad de Farmacia.

10 ACHA (1933), p. 2.

11 Real decreto reformando los estudios de farmacia, de 24 de septiembre de 1886. Publicado en Gaceta de Madrid, 25 de septiembre de 1886, n 268, p. 909-910.

12 Real decreto modificando los estudios de la Facultad de Farmacia, de 31 de julio de 1900. Publicado en Gaceta de Madrid, 3 de agosto de 1900, n²15, p.474. 


\section{LA LEGISLACIÓN Y LA INVESTIGACIÓN CIENTÍFICA}

Además de los planes de estudios publicados en el primer tercio del siglo $\mathrm{XX}$, otras leyes se ocuparon de reglamentar los aspectos relativos a la investigación científica en el ámbito universitario. Desde la primera década del siglo hasta el comienzo de la guerra civil, en 1936, se sucedieron una serie de disposiciones que creaban una red de instituciones y programas de becas y ayudas a la investigación, que tuvieron como consecuencia un desarrollo notable de la experimentación científica en las facultades españolas.

Una de las primeras reglamentaciones fomentando la salida de universitarios a otros países para completar allí su formación, se produjo recién estrenado el siglo XX. El veinte de julio de 1901 se publicaba en la Gaceta de Madrid $^{13}$ la creación de unas pensiones - becas - para ampliar estudios en el extranjero destinadas a «los alumnos que hayan dado mayores pruebas de capacidad y aprovechamiento». Era concedida una por año para cada Facultad y estaban dotadas con 4.000 pesetas abonables por mes vencido. El estudiante que la disfrutase tan sólo tendría que presentar un certificado del cónsul español en el correspondiente país acreditando su residencia en el mismo. Se incluían en la beca los gastos de ida y vuelta con billete de segunda clase. Tras finalizar la estancia debía ser entregada al decano de la Facultad una memoria que resumiese el trabajo realizado en el extranjero. Todas las becas podían ser prorrogadas por un año si el alumno era merecedor de dicha gracia.

No tenemos constancia de que ningún alumno de la Facultad de Farmacia de Santiago disfrutase una de estas becas, si bien pocos años después serían varios los que seguirían el camino de la formación científica en el extranjero. Sin embargo, en 1905 el Ministerio de Instrucción Pública y Bellas Artes concedió una subvención de 3.000 pesetas para que el catedrático numerario de Química Inorgánica de la Facultad de Farmacia de Santiago, José Deulofeu y Poch ampliase estudios de Bacteriología en Francia y Bélgica ${ }^{14}$. Este fue un hecho singular que sin duda introdujo conceptos vanguardistas acerca de la investigación científica y, sobre todo, de la docencia experimental, tal y como se desprende de la memoria entregada por el profesor Deulofeu al Ministerio a su regreso a España ${ }^{15}$.

13 Real Decreto de 18 de julio de 1901 . Publicado en la Gaceta de Madrid de 20 de julio de 1901, no 201. pp.295-296.

14 Real Decreto de 15 de junio de 1905. Publicado en la Gaceta de Madrid de 22 de junio de $1905, n^{\circ} 173$, p. 1175.

15 Deuloféu Y Poch, J. (1906) Consideraciones acerca de la enseñanza de las Ciencias 
En este interesante documento, el catedrático de Química Inorgánica da cuenta del trabajo realizado durante su estancia en la Escuela de Farmacia y el Instituto Pasteur de París, así como los Institutos Pasteur de Lille y Bruselas, Instituto de higiene y Bacteriología de Gante y el Instituto de Bacteriología y Escuela de Química Industrial de Lyon. Todos estos centros representaban la vanguardia europea en la investigación microbiológica de la época. El profesor Deulofeu realizó en esta memoria una evaluación comparativa entre la enseñanza que él recibió durante su estancia, esencialmente práctica y con los medios materiales y humanos necesarios para tal fin, con la triste realidad que en aquel momento representaba la docencia universitaria en ciencias experimentales en una Facultad de provincias como la de Farmacia de Santiago. En 1906, los laboratorios de Fonseca carecían de instalaciones de gas y electricidad $^{16}$, a diferencia de la Universidad Central de Madrid, que aunque remotamente, podría equipararse a otros centros europeos.

Pero sin duda, lo más importante de esta memoria, es el espíritu crítico del autor aplicado a todos los aspectos que él, como hombre de Ciencia, consideraba que debían ser reformados para poder desarrollar, dentro de la Universidad española, trabajos científicos de calidad. Sugiere que desde la administración pública se promueva el que las reformas publicadas en la Gaceta de Madrid tengan una aplicación real, que se incremente el personal auxiliar en los laboratorios universitarios, que se invierta en material y aparatos de calidad para realizar trabajos de investigación, que se seleccione el alumnado mediante exámenes de ingreso y que se trate con menor indulgencia a los alumnos cuyo interés y aprovechamiento no fuese el adecuado. Se trataba de mejorar el nivel de trabajo, esfuerzo y mérito académico de los estudiantes, a fin de que

\footnotetext{
«esas medianías, imposibilitadas de arrastrarse curso tras curso por las aulas universitarias, podrían dedicar su actividad a la agricultura, a la industria, o a las artes, en donde sus servicios serían más provechosos para la Patria, y dejarían de constituir ese lastre enojoso que usurpa los puestos al mérito real y positivo.» ${ }^{17}$
}

En su afán de contribuir a la mejora de la investigación científica en España, plantea una serie de recomendaciones para mejorar las pensiones como la que él mismo había disfrutado, como

\footnotetext{
experimentales y muy en particular de la Bacteriología: Memoria presentada al Gobierno de S.M. en cumplimiento de lo que dispone el R.D. de 8 de Mayo de 1903. Santiago de Compostela.

16 Deuloféu Y PoCH, J. (1906), p.32.

17 Deuloféu Y Poch, J. (1906), p. 42.
} 
«crear en el Ministerio de Instrucción pública un centro informativo, puramente técnico, con la misión de facilitar al pensionado que lo solicitara toda suerte de datos relacionados con la valía profesional y didáctica de profesores extranjeros [...] indicando los estudios a que principalmente se dedican, importe de matrículas, etc. [...] con estos datos, el pensionado podría hacer su composición de lugar antes de emprender el viaje.»

También propuso aumentar la cuantía económica de las pensiones debido a los altos precios de matrícula de los cursos en el extranjero, así como los gastos en material necesarios para desarrollar los trabajos de investigación.

En 1907 se creó la Junta para la Ampliación de Estudios e Investigaciones Científicas $\left(\mathrm{JAE}^{18 \cdot 19}\right)$, aunque el reglamento no se aprobó hasta seis meses más tarde ${ }^{20}$. Quedaba así oficializada la investigación científica en el país, que desde ese momento contaría con la ayuda de la Administración para regular aspectos tales como la dotación económica y en infraestructuras. Con una clara visión aperturista, mirando hacia Europa y promoviendo el contacto con la vanguardia científica, es un hecho ampliamente respaldado el que la JAE consiguió elevar, en apenas tres décadas, la investigación española hasta un notable nivel de producción de trabajos y, sobre todo, de personal investigador. Así quedó dicho en el preámbulo de la Real Orden por el Ministro de Instrucción Pública Amalio Gimeno y Cabañas:

«El más importante grupo de mejoras que pueden llevarse a la instrucción pública es aquel que tiende por todos los medios posibles a formar el personal docente futuro y dar al actual medios y facilidades para seguir de cerca el movimiento científico y pedagógico de las naciones más cultas, tomando parte en él con positivo aprovechamiento. [...] El pueblo que se aísla, se estaciona y se descompone.»

Sin embargo, la JAE presentaba algunas carencias en sus propósitos y funciones. La mayoría de los laboratorios donde se realizaban las investigaciones se localizaban en Madrid ${ }^{21}$, por lo que el resto de las provincias españolas se

18 Real Orden de 11 de enero de 1907, publicada en la Gaceta de Madrid de 15 de enero de $1907, \mathrm{n}^{\circ} 15, \mathrm{pp} .165-167$.

19 Para una mayor información acerca de la Junta para la Ampliación de Estudios recomendamos la lectura del Dossier: en el centenario de la JAE publicado en Asclepio, Vol LIX, No 2 (2007).

20 Real Orden de 16 de junio de 1907. publicada en la Gaceta de Madrid de 22 de julio de 1907, no 173, pp. 1139-1140.

21 Formentín IbÁÑEZ, J. y RodríGuez Fraile, E. (2001), La Fundación Nacional para Investigaciones Cientificas (1931-1939), Madrid, pp. 15 y 16. 
veía claramente en desventaja. Este centralismo se vio atenuado en Galicia por la creación de la Misión Biológica de Galicia (1921), el Seminario de Estudios Gallegos (1923) y la Estación de Biología Marina de Marín (1932) ${ }^{22}$.

En un principio, toda la infraestructura de la JAE se construyó al margen de las universidades puesto que, como hemos apuntado anteriormente, la institución universitaria era vista como un organismo anclado en el pasado y en las enseñanzas poco vanguardistas de los catedráticos decimonónicos. No obstante, a medida que se fue consolidando el trabajo investigador, la JAE comenzó a interaccionar con la Universidad, fundamentalmente con la Universidad Central pero también con las demás universidades periféricas, en busca de activos investigadores con los que conformar un sistema sólido que igualase la Ciencia española con el nivel alcanzado en el resto de Europa. En estas relaciones con la Universidad jugó un papel fundamental el programa de formación de investigadores y docentes organizado por la JAE que permitió la formación en los laboratorios de la Universidad Central o en el extranjero del nuevo personal universitario mediante becas y pensiones de estudios. Siete de las ciento veintisiete becas que otorgó la JAE a investigadores en todos los ámbitos de la incipiente ciencia en Galicia, fueron disfrutadas por profesores y alumnos de la Facultad de Farmacia de Santiago de Compostela ${ }^{23}$. Sin duda un número nada despreciable si tenemos en cuenta las reducidas dimensiones de la facultad y el hecho de pertenecer a una universidad periférica.

Como complemento a las iniciativas realizadas desde la administración para elevar al nivel que le correspondía a la investigación científica española ${ }^{24}$,

22 La Misión Biológica de Galicia se encargó de la investigación en genética vegetal y animal con el fin de mejorar especies de plantas, como el maíz o el castaño, y de ganado, vacuno y porcino. El Seminario de Estudios Gallegos nació de la preocupación de varios estudiantes de la universidad de Santiago por la cultura y el entorno de Galicia, organizando excursiones científicas y estudios acerca de las diferentes comarcas de la región. Por otra parte, la Estación de Biología Marina de Marín se creó como centro dependiente del Museo Nacional de Ciencias Naturales para que los alumnos de Madrid completasen su formación con conocimientos en Biología Marina. Para más información acerca de estas instituciones y de la presencia de la JAE en Galicia, véase FragA VÁzQueZ, X.A. (ed.) (2007) Redes de coñecemento: a Junta para Ampliación de Estudios e a ciencia en Galicia, Madrid, CSIC.

23 Fraga VÁZQUEZ, X.A. (ed.) (2007).

24 En el preámbulo de la Ley se relatan los órganos de fomento de la investigación existentes en otros países (Bélgica, Alemania, Inglaterra, Rusia, EE.UU., Francia, Italia y Portugal) con la intención de compararlos con los existentes en nuestro país. Se recogen datos oficiales sobre ellos, haciendo especial hincapié en la dotación económica destinada a material para laboratorios y proyectos científicos. 
el 13 de julio de 1931 se firmó un Decreto, ratificado como Ley el 5 de diciembre del mismo año por el que se constituyó la Fundación Nacional para Investigaciones Científicas y Ensayos de Reforma que comenzó a recibir subvenciones de los presupuestos del Estado al año siguiente ${ }^{25}$. En su obra acerca de la Fundación Nacional ${ }^{26}$, el doctor Formentín Ibáñez señala cuatro puntos principales en los que esta nueva institución vino a complementar a la JAE:

- Relacionar la investigación con la industria y la empresa privada, de manera que se lograse un desarrollo científico y tecnológico a partir de los medios y el personal que éstas proporcionasen.

- Crear laboratorios en las diferentes universidades españolas.

- Descentralizar la infraestructura para la investigación, concentrada en Madrid desde la creación de la JAE.

- Cooperar con cualquier entidad ministerial, municipal o ciudadana que estuviese interesada.

En la exposición previa al desarrollo de la Ley, aunque se reconoce el excelente trabajo realizado por la JAE, se señalan estas carencias y a la Fundación Nacional como uno de los primeros intentos mundiales por institucionalizar la investigación científica. Resulta curioso ver cómo se hablaba en aquel momento de la «emigración de cerebros» y de la necesidad de terminar con ese fenómeno - algo tan de actualidad en nuestros tiempos-, puesto que después de alcanzar una buena formación, los investigadores no encontraban un lugar en el que poder aplicarla. De este modo, se pretendía modernizar la investigación científica e integrarla en la sociedad como una herramienta útil para su desarrollo económico.

Desgraciadamente, los buenos propósitos de la Fundación Nacional se vieron frustrados el 18 de julio de 1936, al comenzar la guerra civil española, sin que apenas llegasen a tener aplicación.

\section{EL PERSONAL INVESTIGADOR}

Como consecuencia de las políticas anteriormente citadas y los cambios realizados, las universidades españolas sufrieron una metamorfosis durante el

25 Ley de 23 de julio de 1932, publicada en la Gaceta de Madrid de 21 de agosto de $1932, n^{\circ} 234$, p. 1362.

26 Formentín IBÁÑEZ, J. y RodríGuez FraILE, E. (2001), p.15 y 16. 
primer tercio del siglo XX en lo que respecta a sus plantillas docentes. Progresivamente, los catedráticos decimonónicos, cuyas clases magistrales excesivamente teóricas, resultaban difíciles de seguir, fueron siendo sustituidos por jóvenes profesores que incitaban a los estudiantes a participar en la clase, a experimentar y a investigar en los laboratorios.

La Facultad de Farmacia de Santiago de Compostela constituyó un excelente ejemplo de este cambio. Se reclamaba, desde la docencia de la Facultad, una formación científica de calidad con el fin de diversificar las salidas profesionales de los estudiantes de Farmacia. En una carta a los alumnos de la Facultad fechada en 191427 y contestando a las preguntas que los estudiantes habían planteado acerca de su futuro dentro de la profesión farmacéutica, el catedrático de Química Orgánica Enrique de Cuenca y Araujo escribía:

«Mientras la botica sea una tienda y el ejercicio profesional no dilate más su campo de acción con la actividad de que es susceptible el rango de conocimiento científico que constituye el potencial universitario, el porvenir será una eterna noche obscura [...] La inspección relacionada con la Higiene en los Ferrocarriles, Hospitales, etc., es otro horizonte que floridamente da sus primicias al Farmacólogo, Químico Analista y Bacteriólogo. Las industrias agrícolas y fábricas de electricidad, pudieran tener muy bien en los Farmacéuticos sus mejores auxiliares».

Del mismo parecer eran los catedráticos César Sobrado Maestro, de Mineralogía y Zoología, Sandalio González Blanco, de Botánica, Miguel M. ${ }^{a}$ Sojo Alonso, de Técnica Física y Análisis Químico, José Deulofeu de Química Inorgánica y Antonio Eleizegui López, de Materia Farmacéutica Vegetal y decano de la facultad. Todos ellos deseaban un mayor reconocimiento de la profesión farmacéutica dentro del ámbito científico. Y sabían que, para ello, la formación del alumnado en los laboratorios de la Facultad era el primer paso.

El fallecimiento o el traslado en estos años de algunos catedráticos (Marcelino Vieites en 1910, Cecilio Neira en 1915, Sandalio González en 1918) no supuso la incorporación inmediata de nuevos profesores puesto que, como era habitual para el ahorro en los presupuestos, se recurrió al método de la «acumulación de cátedra» lo que significaba que un catedrático podía encargarse de la docencia de otra cátedra vacante por un tiempo indeterminado, percibiendo una cantidad de dinero adicional, siempre inferior a un salario completo. Tan solo Enrique de Cuenca sustituyó a Cecilio Neira al frente de la cátedra de Farmacia Práctica. Esta situación llevó a que, en el curso 1927-1928, tan solo impartie-

27 Cuenca Y Araujo, E. (1914), La regeneración de la profesión farmacéutica, Revista de Farmacia, 2, pp. 66-69. 
ron docencia cinco catedráticos en la Facultad de Farmacia. De las dos cátedras vacantes se ocupaban los cuatro profesores auxiliares que entonces trabajaban en Fonseca.

El sistema de acceso a las cátedras universitarias se basaba en una oposición que tenía lugar en Madrid y a la que podían concurrir profesores de toda España. Este hecho dio lugar a lo que se llamó «catedráticos de ida y vuelta». Desde el gobierno se promovía el traslado de catedráticos entre las distintas facultades y era un hecho frecuente el que los profesores más jóvenes accediesen a cualquier plaza disponible en las cuatro facultades de Farmacia con la intención de conseguir un traslado a la Universidad de partida o a la Universidad Central. Esta última solía ser el destino más codiciado.

En el caso de Santiago, hubo ocho catedráticos, de los treinta que impartieron docencia en la Facultad a lo largo de este periodo, que tras ganar la oposición estuvieron en la facultad tres o cuatro años para trasladarse a continuación. Estos cortos periodos de permanencia no favorecían la consolidación del trabajo de investigación ni la creación de una escuela de profesoresinvestigadores. El caso más llamativo lo protagonizó el catedrático de Botánica, Cayetano Cortés Latorre, quien permaneció en la Facultad del 2 de diciembre de 1922 al 1 de enero de 1923, fecha en que tomó posesión de su nueva cátedra en la Universidad de Barcelona.

Otros profesores, sin embargo, aunque formados en diversas facultades de Farmacia españolas, recalaron permanentemente en Santiago, como César Sobrado Maestro, Miguel Ma Sojo Alonso o José Deulofeu y Poch, lo que permitió una implicación personal en el trabajo de las cátedras que ocupaban.

La escuela compostelana también comenzó a dar sus propios frutos en estos primeros años. Como ya se ha señalado, desde que se creara la JAE, que fomentaba la investigación y favorecía la posibilidad de que los profesores recibiesen formación en el extranjero, varios de los jóvenes profesores de farmacia realizaron estudios en prestigiosos centros investigadores fuera del país. Así, Ricardo Montequi realizó una estancia de un año en Burdeos (1916) en el laboratorio de Farmacia Química del profesor Labat y de Química Biológica con el profesor Degines ${ }^{28}$, y tres años en el laboratorio de Análisis Químico de la en Madrid con el profesor José Casares Gil; Carlos Puente Sánchez estudió la síntesis de medicamentos orgánicos durante una estancia en el Instituto Pasteur de París (1921) y asistió en Madrid a los cursos de Er-

28 Archivo Histórico Universitario de Santiago, Expedientes personales profesores, Legajo 878 . 
nest Fourneau ${ }^{29}$ organizados por la JAE; Francisco Giral González estudió Química Orgánica en Alemania con Richard Kuhn ${ }^{30}$ (1934) y en Inglaterra (1935) y Aniceto Charro Arias, repitiendo la experiencia de Montequi realizó estancias en Madrid y Burdeos (1926) ${ }^{31}$.

El relevo generacional fue muy importante en un momento de la historia de los estudios de Farmacia en el que era preciso aplicar modificaciones sustanciales en la didáctica. Entre las figuras decisivas para el cambio destacó en primer lugar el catedrático de Química Inorgánica Aplicada a la Farmacia, José Deulofeu y Poch, quien en 1902 llegó desde la Universidad de Barcelona. Permaneció en Santiago veinticuatro años, durante los cuales impartió útiles cursillos de Química Biológica y de análisis de orina, conocimientos adquiridos en Bélgica y en el Instituto Pasteur de París en donde realizó una estancia becado por el Ministerio de Instrucción Pública. Este catedrático se expresaba así en 1914 a propósito de la docencia experimental:

«Es condición indispensable que el título de Farmacéutico que conceden nuestras universidades represente algo positivo; no basta con tener algunas ideas mejor o peor cimentadas; es necesario saber hacer, y hemos de confesar, aun cuando el rubor invada nuestro rostro que se sale de la Universidad sabiendo poco o no sabiendo hacer nada. El día que nuestras facultades de Farmacia cuenten con Laboratorios apropiados y con personal suficiente, así en número como en aptitud, para poder dar una enseñanza eminentemente práctica, única y provechosa, $[\ldots]$ se aspirará a otra lucha más noble basada en la actividad y en los conocimientos de cada uno» ${ }^{32}$.

La nueva generación de docentes aportó, con su juventud y su formación investigadora, nuevos aires a la vetusta facultad, al darles al trabajo en el la-

29 El profesor Ernest Fourneau (1872-1949) desarrolló su labor en investigación de medicamentos como jefe del laboratorio de química terapéutica en el Instituto Pasteur de París. En 1917 impartió un curso de química farmacéutica en el laboratorio de la facultad de Farmacia de Madrid. Los trabajos de prácticas del curso se realizaron en el laboratorio de química de la $J A E$ dirigido por Casares Gil, y con sus resultados se publicó la obra FouRNEAU, E. y MADInaveitia, A. (1921) Síntesis de medicamentos orgánicos, Madrid, Calpe. El prólogo fue escrito por Rodríguez Carracido.

30 Richard Kuhn (1900-1967) fue profesor en la Universidad de Munich y jefe del departamento de química en el Kaiser Wilhem Institut für medizinische Forschung de Heidelberg. Francisco Giral publicó varios trabajos con él acerca de la vitamina A y los carotenoides. Obtuvo el premio Nobel de Química en 1938.

31 AHUS, Expedientes Docentes, Torre 150-1.

32 Deulofeu Y Poch, J. (1914) Carta dirigida a los alumnos de la facultad de Farmacia de Santiago. Revista de Farmacia, 2, 73-75. 
boratorio y a la investigación científica la importancia que hasta entonces no habían tenido.

\section{LA DOTACIÓN MATERIAL DE LAS CÁTEDRAS}

En el año 1917 el catedrático de Materia Farmacéutica Vegetal, Antonio Eleizegui López, realizó un inventario ${ }^{33}$ del material de investigación existente en los laboratorios de Fonseca. La dotación material de las cátedras, atendiendo a esta enumeración del profesor Eleizegui, parecía ser suficiente para los trabajos realizados, si bien se quejaban de que era una «lástima grande que los otros dos factores indispensables para las enseñanzas prácticas, profesorado auxiliar y locales, sean hoy, tan escaso el primero y tan reducido el segundo».

En la cátedra de Técnica Física y Análisis Químico se disponía

«además de todos los instrumentos y aparatos para la pulverización, disolución, filtración y destilación, de aparatos de medida y peso, de gran precisión: un palmer, un goniómetro de reflexión, pipetas, buretas automáticas, balanzas de precisión (Sartorius) y de semiprecisión (que aprecia medio miligramo), con sus correspondientes cajas de pesas»».

En la cátedra de Farmacia Práctica, se destacaban los trabajos del ya fallecido profesor Cecilio Neira Núñez. Había en este laboratorio un aparato Welt para la preparación de aguas carbónicas artificiales, una lámpara de cuarzo de vapor de mercurio, un alambique de Soubeirán para la obtención de esencias y aguas destiladas, una prensa hidráulica Altmann, un aparato para comprimidos, un porfirizador de bolas, una emulsionadora movida por energía eléctrica, un aparato de Egrot para obtención de extractos en el vacío, un automatizador Giraud y estufas.

El jardín botánico servía a la cátedra de Botánica con sus plantas medicinales indígenas y algunas exóticas, además de un herbario compuesto de 700 especies. Asimismo, se disponía de 192 modelos de anatomía botánica «escrupulosamente hechos por las mejores casas alemanas y francesas», seis microscopios - entre ellos el modelo panorámico de la casa Reichert diseñado por el propio profesor Eleizegui-, un horno Pasteur, una estufa Asomwal y un autoclave.

33 Eleizegui López, A. (1917) Facultad de Farmacia de Santiago. Revista de Farmacia, 2(2), $37-42$. 
En la cátedra de Materia Farmacéutica Vegetal había una colección antigua de partes y productos de las plantas medicinales, otra más reciente de la casa Merck, cinco microtomos «de los mejores modelos», un aparato Reichert de proyección y un epidiascopio, que permitía proyectar no sólo las diapositivas, sino también directamente la imagen del material vegetal colocado sobre la platina. También se disponía de un aparato de microfotografía «cuya utilidad para el estudio es incalculable».

Mineralogía y Zoología exhibía setenta y cinco formas cristalográficas realizadas en cristal, cien talladas en madera, un esclerómetro, un aparato para determinar la elasticidad de los minerales, un refractómetro, un microscopio petrográfico, un aparato de Senarmón para determinar la conductibilidad térmica de los cristales, dos estuches de Platner, etc. Además, poseía una colección de más de mil ejemplares de unas quinientas especies animales, así como figuras plásticas de las principales clases de vertebrados e invertebrados.

Todo este abundante y moderno material fue adquirido, en su mayor parte, con la financiación derivada de los derechos de matrícula que cada año abonaban los alumnos. Las tarifas se incrementaban anualmente lo que provocaba las protestas y las huelgas de los estudiantes. De todos modos, los profesores estaban orgullosos ${ }^{34}$ de la inversión de este presupuesto en material moderno y sofisticado, que permitía realizar trabajos científicos adecuados en el campo de la investigación y el desarrollo de nuevos medicamentos.

\section{LA DIVULGACIÓN DE LA ACTIVIDAD CIENTÍFICA}

En España, país periférico en cuanto a investigación, el proceso de divulgación científica que implica la creación de revistas de carácter científico, se produjo con mayor retraso que en los países de vanguardia. A pesar de ello, a principios del siglo XX algunas publicaciones especializadas como «La Farmacia Española», «La farmacia moderna», «la Farmacia Nueva», «El monitor de la Farmacia y la Terapéutica»o «El restaurador farmacéutico» realizaban una tarea mixta de divulgación profesional y divulgación científica, recogiendo la mayor parte de los trabajos realizados en las facultades españolas. Una revisión de estas colecciones proporciona una valoración bastante acertada de la evolución de la investigación y desarrollo de fármacos en la Facultad de Farmacia de Santiago de Compostela, aunque sin duda ha de tenerse en cuenta que a comienzos del siglo XX era habitual que algunos de los trabajos realizados no

34 Eleizegui López, A. (1917). 
fuesen publicados o simplemente de extraviasen, especialmente si el catedrático responsable era poco escrupuloso con el orden dentro del laboratorio.

Como se deriva de la revisión de los artículos de investigación publicados por el personal de la Facultad de Farmacia de Santiago de Compostela entre los años 1900 y 1936, a pesar de que la Facultad estaba estructurada en cátedras que se encargaban de la docencia de las asignaturas correspondientes, la investigación en medicamentos se realizaba de manera interdisciplinar. Este hecho, que fue una constante en los primeros años, se difumina a medida que se impone la especialización.

\subsection{La Revista de Farmacia}

En el año 1913 comenzó a editarse en la Facultad de Farmacia de Santiago una revista mensual de divulgación científica e información profesional llamada Revista de Farmacia. En ella se publicaban trabajos de investigación originales ${ }^{35}$ y se reproducían o traducían artículos publicados en otras revistas. Los temas tratados eran aquellos de interés para la clase farmacéutica, tal y como se explicaba en su primer número en el que se establecían las normas editoriales de la publicación:

La Revista de Farmacia se publicará una vez cada mes en números de las mismas páginas que el presente y con los grabados que sean necesarios para ilustrar el texto. Cada uno de los números estará distribuido en las secciones siguientes:

Primera sección.- TRABAJOS ORIGINALES: En esta sección, para la cual contamos con la valiosa colaboración de distinguidos Catedráticos de Farmacia y de Ciencias, Farmacéuticos militares y Doctores y Licenciados en Farmacia, se insertarán, por lo menos, dos artículos inéditos de ciencias aplicadas a la Farmacia.

Segunda sección.- MISCELÁNEA CIENTÍFICA: A fin de seguir el movimiento científico se destinará esta sección a estudiar en ella, en la forma más concisa posible, las nuevas substancias introducidas en el uso médico, los materiales farmacéuticos hasta ahora no empleados y que actualmente se preconicen como medicinales, los programas de los Congresos farmacéuticos, los temas desarrollados en los que se celebraron, la descripción de fábricas de productos químicos y farmacéuticos, y, en general, todas las noticias de carácter científico cuyo conocimiento pueda interesar al farmacéutico.

Tercera sección.- BIBLIOGRAFÍA: El estudio y juicio crítico de las obras, monografías y toda clase de publicaciones de carácter científico y farmacéutico se insertará en esta sección.

35 La editorial de la revista se comprometía a publicar al menos dos trabajos inéditos mensualmente. 
Cuarta sección.- NOTICIAS: Las noticias que, sin ser de índole científica, se refieran a los farmacéuticos, tendrán cabida aquí.

Quinta sección.- BOLETÍN COMERCIAL: La importante casa Merck de Darmstadt nos remitirá mensualmente para su publicación, la relación de precio de los principales materiales empleados en la preparación de los medicamentos, y con esta relación y otros datos económicos se formará esta sección.

Al final de cada número se anunciarán las farmacias, regencias y dependencias vacantes o que se desean adquirir.

Cuando por la importancia de algunos artículos publicados en Revistas nacionales o extranjeras hayan de insertarse íntegros, aparecerán, con indicación de su origen, a continuación de los trabajos originales, en una sección que titularemos DE OTRAS REVISTAS.

Se trataba de una publicación que, junto Galicia Médica (Revista de medicina clínica y experimental), editada por profesores de la Facultad de Medicina compostelana, era pionera dentro de la Universidad de Santiago de Compostela, puesto que eran muy escasas las revistas de divulgación científica editadas por las facultades universitarias ${ }^{36}$.

La Revista de Farmacia llegó incluso a publicarse en Latinoamérica, en donde contaba con un representante, el doctor en farmacia Marcial Méndez, afincado en Buenos Aires. La publicidad que hacía la Revista de Farmacia subrayaba las siguientes ventajas:

«Publica en todos sus números trabajos originales de los mejores escritores técnicos españoles sigue el movimiento científico, traduciendo y resumiendo lo más notable de cuanto se escribe en el extranjero; hace el juicio crítico de las obras científicas; contesta a cuantas consultas de carácter científico o administrativo se le hagan; publica en pliegos separados, y con numeración independiente, tratados prácticos originales, de interés profesional; se encarga de pedir y hacer llegar a poder de los suscriptores todas las obras nacionales y extranjeras que deseen, sin recargo alguno y enviando a cobrar su importe al domicilio del suscriptor».

En la sección TRABAJOS ORIGINALES $S^{37}$ publicaban sus artículos los profesores de la Facultad, alumnos, farmacéuticos licenciados, farmacéuticos milita-

36 GurriarÁn Rodríguez, R. (2003) A investigación cientifica en Galicia (1900-1940): Institucións, redes formativas e carreiras académicas. A ruptura de Guerra Civil. Tesis doctoral. Santiago de Compostela, pag. 432.

37 Debemos señalar que en esta sección no todos los trabajos publicados eran de investigación científica experimental, ya que en ocasiones se incluían artículos de opinión acerca de un tema de actualidad que afectase a la profesión farmacéutica o trabajos relativos a la Historia de la Farmacia. 
res $^{38}$ y doctores en Farmacia. Como se deriva de la observación de la figura 1, las aportaciones del personal de la Facultad fueron siempre importantes, pero no cabe duda de que la edición de una revista de ciencias farmacéuticas supuso una interesante forma de divulgación para los trabajos científicos que se estaban realizando en los laboratorios de otras facultades españolas y oficinas de farmacia.

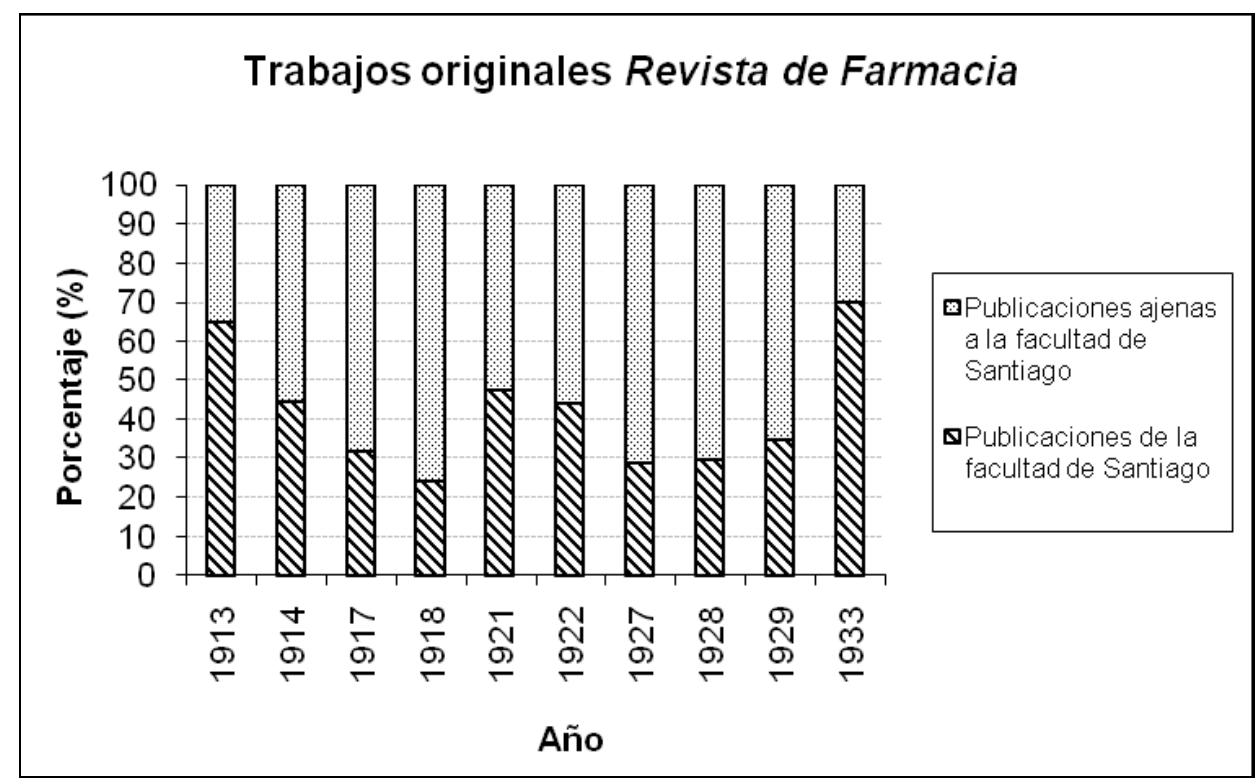

Figura 1. Trabajos de investigación científica publicados en la Revista de Farmacia entre 1913 y 1933

La publicación sirvió, entre 1913 y 1936, de plataforma para la divulgación de la incipiente tarea investigadora que comenzaba a realizarse en los laboratorios de Fonseca, pero además de los trabajos originales, la Revista de Farmacia publicaba artículos de revisión y se hacía eco de los trabajos científicos de carácter farmacéutico que se publicaban en las diferentes revistas

38 El profesor Máiz Eleizegui había ingresado en el cuerpo de Farmacia Militar en 1909, y su relación con otros farmacéuticos militares hizo posible que publicasen trabajos de investigación en la Revista de Farmacia. MÁiz Eleizegui, L. (1961) Historia de la Enseñanza de Farmacia en Santiago (Cien años de vida de la Facultad) 1857-1957, A Coruña, p. 137. 
científicas europeas, lo que le permitía a los investigadores mantener actualizados sus conocimientos.

El análisis de la distribución de los trabajos publicados en función de las distintas disciplinas (Figura 2) proporciona una valiosa información acerca de las líneas de investigación desarrolladas en este centro, aunque como ya se ha señalado, el carácter pluridisciplinar de la investigación que se desarrollaba, hace difícil establecer fronteras entre unas líneas de investigación y otras. Da la impresión de que, en la mayoría de los casos, el trabajo se componía de diferentes estudios realizados en cada momento, sin relación entre ellos.

El número de trabajos realizados por cada cátedra se refleja en la figura 2.

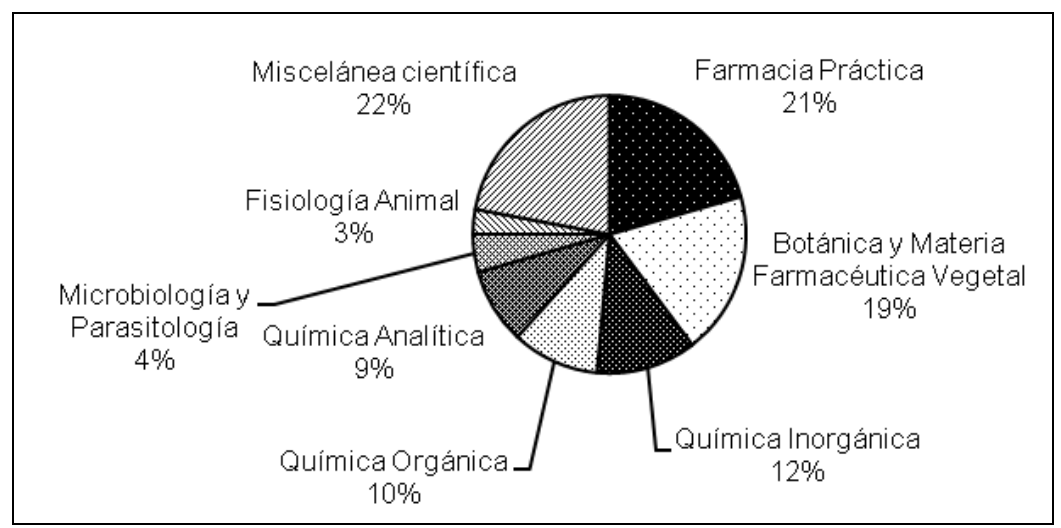

Figura 2. Número de trabajos realizados clasificados por temas

\subsection{Trabajos de investigación ${ }^{39}$}

\subsubsection{Farmacia Práctica}

En la cátedra de Farmacia Práctica comenzaron realizándose trabajos de investigación bajo la tutela del profesor Cecilio Neira. Varios de los trabajos publicados están firmados por alumnos, ya que se distinguió por ser un cate-

39 Para el análisis pormenorizado de estos trabajos, estudiaremos las cuatro cátedras con mayor número de publicaciones y en las que se observa una línea de investigación más definida: Farmacia Práctica, Química Orgánica, Botánica y Materia farmacéutica Vegetal y Química analítica, siendo las dos primeras las que contaban con grupos investigadores más consolidados. 
drático preocupado por acercar a los estudiantes al laboratorio. Puede afirmarse que fue un grupo de trabajo consolidado durante los años que el profesor Neira estuvo al frente de la cátedra, y que continuó su labor cuando en 1918 le relevó en el cargo el doctor Carlos Puente Sánchez, defensor de la enseñanza activa y de la investigación científica en la Universidad. El espíritu investigador del equipo y el esfuerzo por situar el trabajo de los desprestigiados farmacéuticos dentro del ámbito científico se refleja en las palabras de uno de sus miembros, José Fernández de la Reguera, en el encabezamiento de un trabajo publicado en la Revista de Farmacia:

«Ofrecer a la consideración de los Farmacéuticos españoles un algo práctico, un algo capaz de demostrar a los que despectivamente nos miran, que no estamos sumidos ni mucho menos en vergonzosa inopia intelectual, ha sido siempre mi mayor aspiración, mi constante estímulo en la observación de aquello susceptible de perfeccionamiento a lo que he dedicado todos mis entusiasmos $[\ldots] . »$

La investigación de este grupo iba dirigida fundamentalmente al desarrollo o mejora de equipos de producción de medicamentos y a la obtención de diversas materias primas, útiles en el desarrollo de formas farmacéuticas.

A modo de ejemplo puede señalarse un artículo ${ }^{40}$ en el que se describe de manera exhaustiva un nuevo aparato emulsionador desarrollado en el laboratorio de farmacia práctica que corregía las deficiencias encontradas en los equipos emulsionadores comercializados; «agitación deficiente o nula o bien una imperfecta adición del aceite en el seno del vehículo emulsionante» y facilitaba la emulsificación de los preparados.

De igual manera, otro artículo del mismo autor ${ }^{41}$ recoge el trabajo previo del doctor Miguel María Sojo Alonso, catedrático de Botánica, en el desarrollo de un nuevo aparato para la determinación de los puntos de fusión y solidificación.

La optimización de los procesos de obtención de distintos componentes utilizados en el desarrollo de medicamentos u otros productos oficinales descritos por diferentes autores, y su valoración, fue también objeto de especial atención por parte de los investigadores de la cátedra de Farmacia Práctica. Por ejemplo, la preparación de polvo de carne ${ }^{42}$, un dietoterápico muy em-

40 Fernández De La Reguera Y Aguilera, J. (1913), Nuevo aparato emulsionador. Revista de Farmacia, 1(1), 15-20.

41 Fernández De La Reguera Y Aguilera, J. (1913), Nuevos aparatos de aplicación en los trabajos de laboratorio. Revista de Farmacia, 1, 134-137.

42 Fernández De La Reguera Y Aguilera, J. (1913), Obtención del polvo de carne. Revista de Farmacia, 1, 45-48. 
pleado en la época o la peptona pepsino-clorhídrica ${ }^{43}$. A través del cálculo del rendimiento del proceso, aspecto de especial importancia si te tiene en cuenta el elevado precio de las materias primas, se evalúa la eficacia de diferentes métodos de desecación de la carne y se selecciona el que permite obtener un material con menor humedad residual, lo que facilita la conservación del producto y por tanto su mayor estabilidad. Investigación científica e innovación tecnológica se entrelazan en este segundo trabajo en el que la desecación se realiza en estufa de aire seco con termómetro de aviso, un ingenioso sistema diseñado con una alarma acústica que se activaba en el momento en que se alcanzaba la temperatura deseada en el interior del equipo.

En lo que respecta a la optimización de los métodos de preparación de productos oficinales destacan los trabajos realizados con los derivados del cornezuelo de centeno ${ }^{44}$ (Claviceps purpurea). Este hongo parásito fue un producto muy relevante en la época ya que constituía la materia prima para la extracción de alcaloides de uso frecuente en terapéutica, como la ergotina o la ergotamina. En Galicia, el cultivo y recolección del cornezuelo supuso una importante fuente de ingresos para gran parte de la población rural durante la primera mitad del siglo XX. Este trabajo del profesor Neira Mosquera versa sobre la obtención, purificación y valoración de la ergotina medicinal.

Las nuevas formas farmacéuticas también fueron objeto de estudio en los trabajos realizados en Fonseca, aunque de manera minoritaria ya que la investigación sobre síntesis de nuevas moléculas con actividad farmacológica ocupaba el papel protagonista. Los estudios sobre inyectables ${ }^{45}$ fueron un ejemplo de esas nuevas formas de dosificación de coste económico elevado, más exigentes a la hora de su preparación en cuanto a condiciones de asepsia.

\subsubsection{Botánica y Materia Farmacéutica Vegetal}

La Botánica y la Materia Farmacéutica Vegetal eran consideradas como «ciencia de las plantas, aplicada a la preparación de medicamentos ${ }^{46} »$. En

43 NeIRA NúÑEZ, C. (1913), Preparación de la peptona pepsino-clorhídrica por el procedimiento de Petit. Revista de Farmacia, 1, 86-94, 88.

44 NeIRA NúÑEZ, C. (1913), Obtención de la ergotina medicinal. Revista de Farmacia, 1, 129-134.

45 Charro Arias, A. (1935), Isotonia e isoionia de los inyectables. El Monitor de la Farmacia y de la Terapéutica, 1.234, 77-79.

46 Eleizegui LóPEZ, A. (1917), cita p.41. 
este campo tuvo una especial relevancia el trabajo del catedrático Antonio Eleizegui López, estrechamente ligado a la microscopía ${ }^{47}$. Muchos de sus artículos versaron sobre el aislamiento de drogas en vegetales para su posterior empleo como medicamentos. A lo largo de las tres primeras décadas del siglo XX fue muy importante todavía la obtención de principios activos de las plantas. El desarrollo de la química sintética era aún incipiente, de modo que resultaba más sencillo - y económico - extraer los principios activos de las plantas, tal como se había venido haciendo desde el siglo anterior, si bien se modernizaron los métodos para mejorar la pureza de los productos y el rendimiento de los procesos. La I Guerra Mundial (1914-1919) planteó para España un grave problema de desabastecimiento de medicamentos industriales procedentes fundamentalmente de Alemania. De este modo, en esos años y en la correspondiente posguerra, los farmacéuticos españoles se afanaron en la búsqueda de principios activos vegetales y en la mejora de los procesos de extracción y purificación.

\subsubsection{Química Analítica}

La aportación de la cátedra de Química Analítica fue relevante en la década de los años 20. Los cursos de Análisis de medicamentos que el profesor Aniceto Charro Arias recibió durante su estancia en la facultad de medicina y farmacia de Burdeos en 1926, dieron como resultado la publicación de tres artículos sobre este tema en la Revista de Farmacia ${ }^{48,49,50}$. Dichos trabajos fueron realizados en el laboratorio del profesor Labat, investigador de reconocido prestigio en el campo de la Farmacia Química.

El catedrático de Química Inorgánica, Ricardo Montequi, también realizó estudios en el campo de la Química Analítica aplicada a las ciencias farmacéuticas $^{51,52}$.

47 Eleizegui desarrolló un modelo de microscopio panorámico que la casa Reichert (Alemania) construyó y comercializó. MAIz Eleizegui, L. (1961), p.69.

48 Charro Arias, A. (1927), Análisis de aguas. Revista de Farmacia, 15, 261-266.

49 Charro Arias, A. (1927), Análisis de orinas. Revista de Farmacia, 15, 293-303.

50 Charro Arias, A. (1928), Análisis de sangre. Revista de Farmacia, 16, 81-93.

51 Montequi, R y De SÁdABA, R. (1931), La investigación del sodio con el reactivo de Streng-Kolthoff, Anales de la Sociedad Española de Física y Química, 29, 255-261.

52 Otero Aenlle, E. y Montequi Y Díaz De Plaza, R. (1935), Contribución al estudio de la determinación del wolframio con oxiquinoleína. Boletín de la Universidad de Santiago, 23, 231-239. 


\subsubsection{Química Orgánica}

El interés por la Química Orgánica aplicada al desarrollo de nuevos medicamentos se despertó de una manera temprana en la Facultad de Farmacia de Santiago. Prueba de ello es el extenso trabajo de revisión publicado en 1914 por el profesor Luís Máiz Eleizeguii ${ }^{53}$ sobre la síntesis químico-orgánica de los compuestos más relevantes, los compuestos hidrocarburados y oxhidrocarburados. A este primer trabajo siguieron otros como el del profesor auxiliar Leopoldo López Pérez ${ }^{54}$ acerca del desarrollo de un método de obtención del antitérmico, acetanilida o antifebrina, mediante un procedimiento sintético a partir de la anilina y el ácido acético. En los agradecimientos del trabajo se hace referencia a la relación que se mantenía entre la Universidad de Santiago y la Universidad Central ya que el trabajo fue verificado en el laboratorio de Química Orgánica de esta Universidad por los doctores Bonet y Bonet y José. R. Carracido.

Los cursillos de síntesis orgánica de medicamentos que impartieron los profesores Charro Arias y Carlos Puente en la década de 1920 y hasta el año 1933 fueron un elemento muy positivo dentro de la investigación y desarrollo de fármacos en Compostela, que contribuyó a fomentar el interés de los alumnos por el trabajo investigador dentro de la cátedra de Química Orgánica. El programa del curso académico 1921-1922 se publicó en la Revista de Farmacia ${ }^{55}$. Se dividía en una parte teórica y otra, mucho más extensa, práctica. La teórica consistía en conferencias públicas, celebradas las mañanas de los miércoles y los sábados en la facultad, centradas en dos temas principales: Farmacodinamia -ideas generales sobre acción de medicamentos, antipiréticos, hipnóticos, antisépticos, arsenicales orgánicos, anestésicos locales - y Métodos de Química Orgánica como halogenación, nitración, oxhidrilación, sulfonación, carboxilación, acilación, saponificación, oxidación y reducción. La parte práctica estaba dirigida a la síntesis de diversos medicamentos, entre ellos la novocaína, la aspirina o el salvarsán.

\section{PARA CONCLUIR}

En el primer tercio del siglo XX, aunque España no formaba parte del grupo de países dinamizadores en ciencia, no por ello se sustraía al importante

53 Máiz Eleizegui, L. (1914), Síntesis químico-orgánica. Revista de Farmacia, 2(1), 1-9.

54 LÓPEZ PÉREZ, L. (1914), Obtención sintética de la acetanilida o antifebrina. Revista de Farmacia, 2(1), 9-14.

55 Revista de Farmacia (1921), 9, 286-288. 
movimiento mundial hacia la universalización de la ciencia. España, país de tradición centralista se unió, en la medida de lo posible, a esa carrera hacia el desarrollo científico que se había iniciado en toda Europa. En este contexto, resulta interesante el estudio de la contribución al desarrollo científico de facultades como la de Farmacia de Santiago, perteneciente a una Universidad periférica, con poca dotación económica, de infraestructuras y de personal.

Las infraestructuras de la Facultad mejoraron de forma sustancial como consecuencia de los incrementos presupuestarios. La mejora no presentaba excesiva dificultad dada la precaria situación en la que se encontraban a comienzos de siglo: ausencia de agua, electricidad y gas.

La legislación relativa a la investigación en las universidades españolas se caracterizó, durante este periodo, por un fomento de la formación del profesorado en centros investigadores de prestigio en los laboratorios de la Universidad Central y del extranjero mediante una política de becas.

El relevo generacional y la incorporación de personal investigador joven y entusiasta fomentaron el desarrollo de la investigación científica en la Facultad de Farmacia de Santiago como se refleja en los trabajos publicados por profesores y alumnos en las revistas especializadas de la época. El trabajo realizado permitió situar a este centro a un nivel digno, dentro del panorama científico español.

La guerra civil supuso la paralización de la actividad docente e investigadora de la facultad de farmacia durante la contienda y la dictadura posterior, una ruptura de las políticas que fomentaban la actividad investigadora. A pesar de ello, los años de la guerra no consiguieron la suspensión de la actividad en la vetusta Fonseca puesto que funcionó como Laboratorio de Farmacia Militar que abastecía de medicamentos al ejército franquista.

Fecha de recepción: 4 de abril de 2008

Fecha de aceptación: 4 de diciembre de 2008 